\title{
VARIACIÓN MORFO-AGRONÓMICA EN ACCESIONES DE Elymus scabrifolius DEL CENTRO-NORTE DE SANTA FE
}

\author{
TOMAS, P. A. ${ }^{1}$; GIAVEDONI, J. A. ${ }^{1}$; ZABALA, J. M. ${ }^{1}$ \& SCHRAUF, G. E. ${ }^{2}$
}

\begin{abstract}
RESUMEN
Elymus scabrifolius es una gramínea perenne nativa de Sudamérica, considerada como potencial recurso forrajero para suelos con limitantes edáficas. Los objetivos de este trabajo fueron analizar la variación genética existente para caracteres agronómicos y determinar patrones de variación fenotípica para 17 accesiones y un cultivar comercial, mediante análisis de varianza y técnicas multivariadas. Los materiales presentaron diferencias significativas para todas las variables agronómicas analizadas. En el análisis de agrupamiento, el cultivar comercial se diferenció claramente de las otras accesiones, las cuales se agruparon formando dos conglomerados según su región de procedencia. Los resultados del Análisis de Componentes Principales fueron concordantes con lo observado en el dendrograma. Los resultados indicaron la existencia de considerable variación genética entre los materiales analizados y sugieren la importancia del origen geográfico tanto en la distribución de dicha variación, como así también en la búsqueda de nueva variación genética para esta especie.

Palabras clave: Agropiro criollo, Elymus scabrifolius, forrajera nativa, patrón de variación, análisis multivariado, recursos genéticos.
\end{abstract}

\section{SUMMARY}

\section{Morpho-agronomic variation in Elymus scabrifolius accessions from centre- north of Santa Fe. \\ Elymus scabrifolius is a perennial South American wheatgrass, considered as a potential forage resource for environments with soil limitations. The aims of this work were to analyze the genetic variation for agronomic traits and determine patterns of phenotypic variation for 17 accessions and one commercial cultivar, by analysis of variance and multivariate analysis. All traits showed significant}

1.- Facultad de Ciencias Agrarias (UNL). Kreder 2805. (3080) Esperanza, provincia de Santa Fe. Email: patomas@fca.unl.edu.ar

2.- Facultad de Agronomía, Universidad de Buenos Aires. San Martín 4453. (1417) Ciudad Autónoma de Buenos Aires, Argentina.

Manuscrito recibido el 30 de noviembre de 2011 y aceptado para su publicación el 3 de marzo de 2012 